\title{
The insulin/Akt pathway controls a specific cell division program that leads to generation of binucleated tetraploid liver cells in rodents
}

\author{
Séverine Celton-Morizur,,1,2 Grégory Merlen,,1,2 Dominique Couton,1,2 \\ Germain Margall-Ducos,,1,2 and Chantal Desdouets ${ }^{1,2}$ \\ ${ }^{1}$ Institut Cochin, Université Paris Descartes, CNRS UMR 8104, Paris, France. 2INSERM U567, Paris, France.
}

\begin{abstract}
The formation of polyploid cells is part of the developmental program of several tissues. During postnatal development, binucleated tetraploid cells arise in the liver, caused by failure in cytokinesis. In this report, we have shown that the initiation of cytokinesis failure events and the subsequent appearance of binucleated tetraploid cells are strictly controlled by the suckling-to-weaning transition in rodents. We found that daily light/dark rhythms and carbohydrate intake did not affect liver tetraploidy. In contrast, impairment of insulin signaling drastically reduced the formation of binucleated tetraploid cells, whereas repeated insulin injections promoted the generation of these liver cells. Furthermore, inhibition of Akt activity decreased the number of cytokinesis failure events, possibly through the mammalian target of rapamycin signaling complex 2 (mTORC2), which indicates that the PI3K/Akt pathway lies downstream of the insulin signal to regulate the tetraploidization process. To our knowledge, these results are the first demonstration in a physiological context that insulin signaling through Akt controls a specific cell division program and leads to the physiologic generation of binucleated tetraploid liver cells.
\end{abstract}

\section{Introduction}

Polyploidy, the state of having greater than a diploid DNA content (tetraploid, octoploid, etc.), is a widespread physiological phenomenon observed particularly in plants, fish, and amphibians (1). Although it is less common in mammals, some tissues - including heart muscle cells, platelet progenitor megakaryocytes, and liver parenchyma - develop a certain degree of polyploidy during their normal life cycle. The appearance of polyploid cells is often associated with late fetal development and coincides with terminal differentiation $(2,3)$. Polyploid cells can be also formed in response to stress and injury, as has been described previously for uterine smooth muscle during pregnancy (4), heart muscle cells and VSMCs during hypertension $(5,6)$, and thyroid cells in hyperthyroidism $(7,8)$. Moreover, in many human carcinomas, cells with polyploid DNA content arise as an early step in tumorigenesis and precede the formation of aneuploid cells $(8,9)$.

Polyploidy is a characteristic feature of mammalian hepatocytes (10). During postnatal growth, the liver undergoes substantial changes, including gradual polyploidization. This process generates the successive appearance of tetraploid and octoploid hepatocytes with 1 (mononucleated; e.g., $4 \mathrm{n}, 8 \mathrm{n}$ ) or 2 nuclei (binucleated; e.g., $2 \times 2 n, 2 \times 4 n$ ). In adults, $70 \%$ of all hepatocytes in rodents and $40 \%$ in humans are tetraploid $(11,12)$. Interestingly, in the adult liver, the generation of polyploid cells can be reinduced following a variety of cellular stressors. Liver regrowth after partial hepatectomy or following oxidative damage and metabolic overload (copper/iron) is associated with a pronounced increase in the proportion of cells that are polyploid (13-16).

Conflict of interest: The authors have declared that no conflict of interest exists.

Nonstandard abbreviations used: FAS, fatty acid synthase; GK, glucokinase; L-PK, liver-pyruvate kinase; mTOR, mammalian target of rapamycin; mTORC, mTOR signaling complex; STZ, streptozotocin.

Citation for this article: J. Clin. Invest. 119:1880-1887 (2009). doi:10.1172/JCI38677.
One fascinating question is how diploid organisms give rise to polyploid cells. In some cell types, such as skeletal muscle cells and osteoclasts, cell fusion is a normal developmentally programmed step and leads to the production of terminally differentiated polyploid cells $(17,18)$. Polyploid cells can also be generated by endoreplication, as has been observed previously in megakaryocytes and vascular smooth muscle cells $(19,20)$; during this specialized cell cycle, cells skip mitosis and proceed to several rounds of DNA replication. Finally, cytokinesis might fail because of a disturbance of cleavage furrow formation. Such cytokinesis failure has been clearly identified during pathological division (9). Using live-cell microscopy, we previously demonstrated that, surprisingly, hepatocytes become polyploid by physiological failure of cytokinesis (21). Diploid hepatocytes undergo mitosis, but do not form a contractile ring. Indeed, cleavage-plane specification is never established, because of deficiencies of actin cytoskeleton reorganization. Consequently, microtubules fail both to contact the cortex and to deliver their molecular signal, preventing localization and activation of RhoA. Therefore, cytokinesis aborts, and a binucleated tetraploid liver cell is generated (22). This specific cell cycle program takes place at weaning. During this period, diploid hepatocytes engage either into a normal cell cycle, giving rise to 2 diploid hepatocytes, or do not complete cytokinesis, and generate a single tetraploid hepatocyte with 2 nuclei (21). The generation of such binucleated tetraploid cells is the crucial step for the establishment of polyploidization during postnatal liver growth. Indeed, if this hepatocyte divides again, mononucleated tetraploid progenies will be generated.

To our knowledge, the cell signaling mechanism that controls the cytokinesis failure program and leads to the generation of a binucleated tetraploid liver cell has not previously been elucidated. In this report, we demonstrate that the PI3K/Akt pathway, downstream from insulin signaling, controlled this specific division program and thus the generation of binucleated tetraploid liver cells. 
A Normal cytokinesis

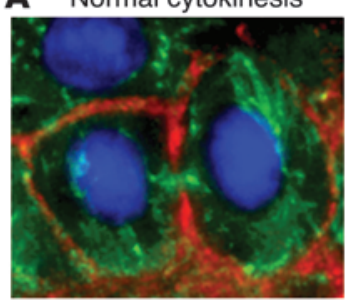

Cytokinesis failure

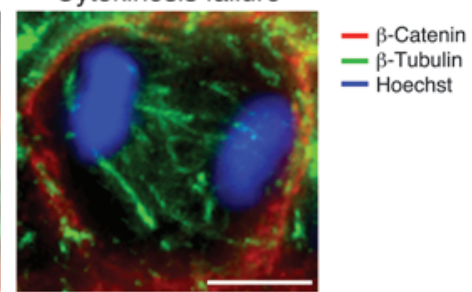

B

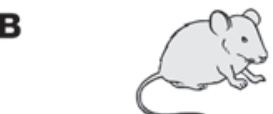

15 days suckling

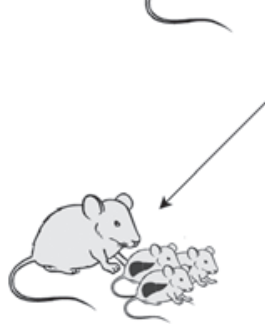

19 days suckling

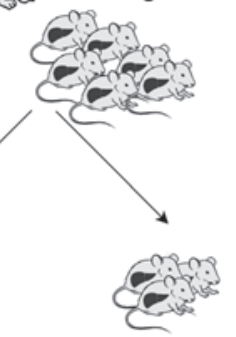

19 days weaned

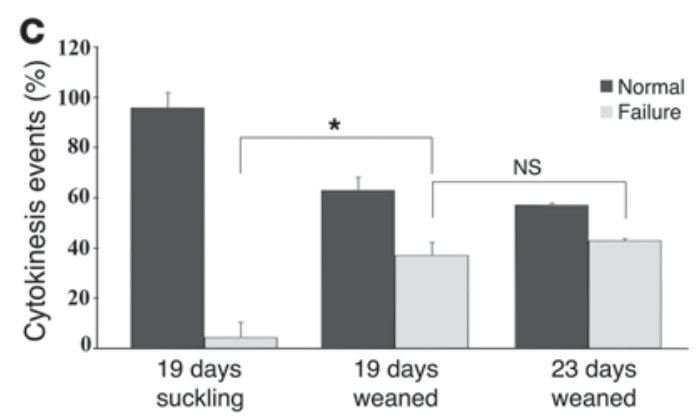

\section{Results}

Generation of binucleated tetraploid hepatocytes is controlled by the suckling-to-weaning transition. We first investigated the involvement of animal weaning in the control of cytokinesis failure events by analyzing mitosis events on tissue liver sections (Figure 1A). When hepatocytes completed cytokinesis, a particular microtubule structure, called midbody, was formed, and the cell adopted a shape characteristic of cleavage furrow ingression. In contrast, when hepatocytes did not complete cytokinesis, microtubule networks were clearly disorganized, and membrane ingression was absent. In rats weaned at age 19 days and fed a high-carbohydrate diet for 4 days (Figure 1B), we observed that a high proportion of dividing hepatocytes did not complete cytokinesis $(43.0 \% \pm 0.8 \%$; Figure $1 \mathrm{C}$ and ref. 22). In rats suckling until 19 days, most hepatocytes completed cytokinesis, whereas in their littermates weaned at age 15 days and fed a high-carbohydrate diet until 19 days, already $36.9 \% \pm 5.2 \%$ of hepatocytes failed to complete cytokinesis (Figure 1C). Thus, the widespread failure of cytokinesis in hepatocytes, and the consequent generation of binucleated tetraploid cells, was controlled by the suckling-to-weaning transition.

Daily rhythms and carbohydrate supply do not affect the tetraploidy process. The suckling-to-weaning transition is associated with modifications of various physiological processes, both nutritional and hormonal (23). Suckling animals are on a low-carbohydrate, high-fat diet (maternal milk) and, after weaning, are shifted to a high-carbohydrate, low-fat diet. In parallel, large modifications

\section{Figure 1}

Cytokinesis failure is induced by the suckling-to-weaning transition. (A) Immunostaining of liver sections with $\beta$-catenin (plasma membrane labeling) and $\beta$-tubulin (microtubule labeling). Images of telophase were visualized as condensed chromatin staining (Hoechst 33342 ). Immunostaining allowed telophases that completed cytokinesis to be distinguished from those that did not. Scale bar: $10 \mu \mathrm{m}$. (B) Left: Rats from the same litter were either weaned at age 15 days and fed a high-carbohydrate diet or left with their mother with no access to solid food. All rats were sacrificed at 19 days of age. Right: Rats from a single litter were weaned at 19 days of age, fed a high-carbohydrate diet, and sacrificed at day 23. (C) Cytokinesis in 19-day suckling and in 19- and 23-day weaned rats as in B. Percentages of normal cytokinesis and cytokinesis failure were calculated by analysis of $\beta$-catenin, $\beta$-tubulin, and Hoechst immunostaining on tissue sections. For each animal, 50 telophases were analyzed. Results are means \pm SEM ( $n=6$ per group). ${ }^{*} P<0.05$.

in the concentration of circulating hormones, such as glucagon and insulin, are observed; respectively, they are high and low during suckling and the reverse after weaning. Furthermore, daily rhythms progressively emerge during this physiological transition. In the liver, DNA synthesis and mitotic index are correlated with the diurnal period $(24,25)$. We therefore tested whether one or several of these parameters control liver tetraploidization.

We first assessed whether modulation of daily rhythms affected the generation of binucleated tetraploid hepatocytes by modifying the daily light/dark cycle for 1 week after weaning (Figure 2A). At this stage of liver development, the tetraploidization process is already strongly engaged (21). We used simultaneous immunolabeling of nuclear and plasma membranes in tissue sections to distinguish between diploid and tetraploid fractions (Figure 2B). Perturbation of daily rhythms did not interfere with the generation of binucleated tetraploid hepatocytes (Figure 2C).

We next tested whether nutritional supply, particularly an increase in the carbohydrate content of the diet, affected the generation of binucleated tetraploid hepatocytes. Rats from the same litter were fed for 6 days with either a high-carbohydrate, low-fat diet or a low-carbohydrate, high-fat diet (Figure 3A). Because long-term high-fat dieting may induce hyperinsulinemia and hyperglycemia (26), we tested whether blood glucose and insulin levels were modified in our conditions of short-term high-fat diet feeding; no significant increase was observed (Figure 3B). In parallel, we analyzed the expression levels of key factors controlling glycolytic and lipogenic pathways, including SREBP-1c, glucokinase (GK), liver-pyruvate kinase (L-PK), and fatty acid synthase (FAS) (27). Under short-term high-fat diet feeding, SREBP-1c and GK expressions were unaffected (Figure 3, C and D), which indicates that the insulin signaling pathway was functional. Conversely, a significant decrease of FAS (insulin and glucose signaling) and L-PK expression (glucose signaling only) was observed in the high-fat diet group (Figure 3, C and $\mathrm{D}$ ), indicating that glucose signaling was impaired. In both diet conditions, we quantified the percentage of diploid and tetraploid hepatocyte populations (see Figure $2 \mathrm{~B}$ ), and found that the different diets did not perturb the generation of binucleated tetraploid hepatocytes (Figure 3E). Together, these data indicate that glucose signaling is not essential for tetraploidization in the liver.

Insulin concentration regulates generation of binucleated tetraploid hepatocytes. To reduce the level of circulating insulin, rats were treated before weaning with streptozotocin (STZ), which selectively destroys pancreatic $\beta$ cells (Figure 4A). STZ-treated rats showed 
A

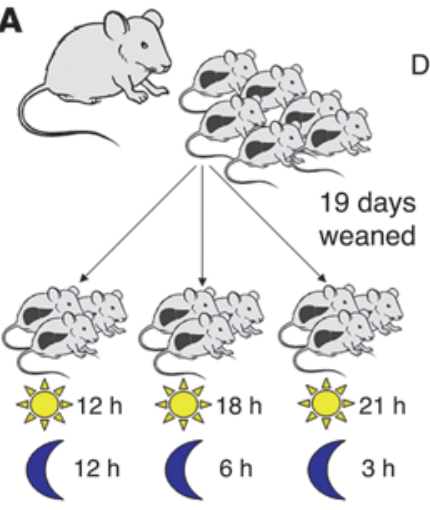

B

Diploid (2n)

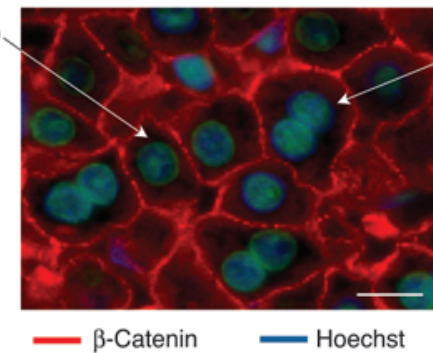

Tetraploid

$(2 \times 2 n)$

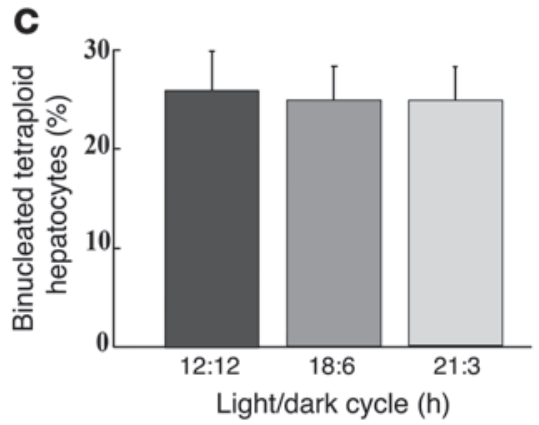

Figure 2

Influence of circadian clock on the tetraploidy process. (A) Rats were weaned at 19 days of age and fed a high-carbohydrate diet; individuals from the same litter were assigned to light/dark cycles of 12:12, 18:6, or 21:3 hours. All rats received a high-carbohydrate diet and were sacrificed at 25 days of age. (B) Image of tissue section after double staining with $\beta$-catenin and Hoechst, illustrating the difference between diploid and tetraploid hepatocytes. Scale bar: $10 \mu \mathrm{m}$. (C) Percent binucleated tetraploid cells was calculated by analysis of $\beta$-catenin/Hoechst immunostaining. Histogram shows mean \pm SEM of 3 independent experiments. A minimum of 2,500 hepatocytes in 8-12 separate, randomly selected fields was analyzed ( $n=5$ per group).

substantially reduced levels of circulating insulin and hyperglycemia 5 days after the end of treatment (Figure 4B). We analyzed the effect of this treatment on the expression of genes regulated by insulin and glucose. STZ treatment downregulated both insulin and glucose pathways, as expected. The expression of SREBP-1c, GK, FAS, and L-PK decreased, as assessed by quantitative realtime PCR and Western blotting (Figure 4, C and D). Moreover, the amount of phospho-Akt (Ser473 residue) was lower in STZ-treated rats than in controls (Figure 4D), again reflecting the impairment of the insulin pathway. Importantly, we demonstrated that in this condition, the binucleated tetraploid hepatocyte content $(4.1 \% \pm 3.7 \%)$ was much lower in STZ-treated rats than in control animals $(19.9 \% \pm 6.6 \%$; Figure $4 \mathrm{E})$. We previously illustrated that by isolating primary hepatocytes during postnatal liver develop-
A

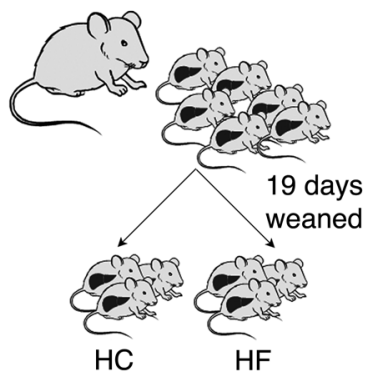

C

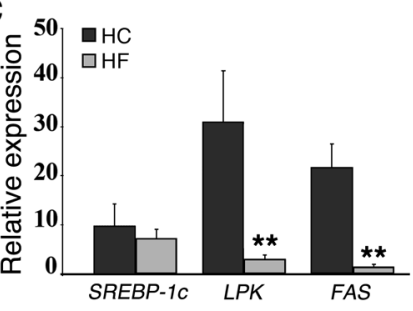

B
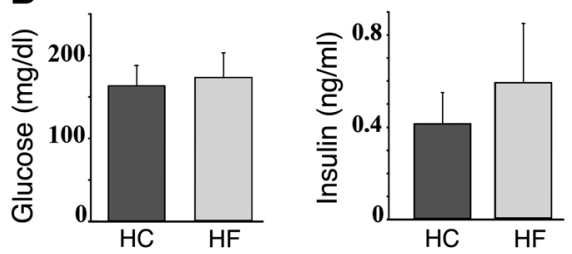

D
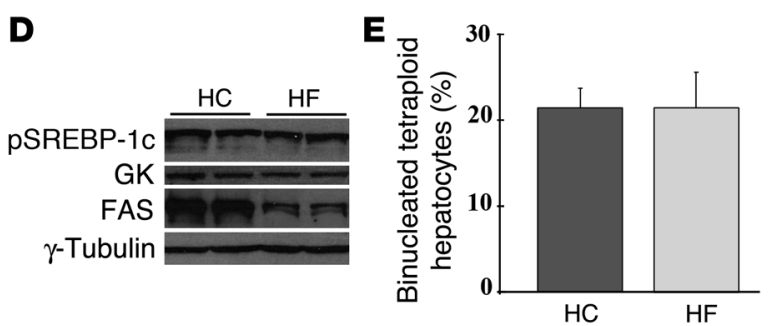

Figure 3

Influence of carbohydrate intake on the tetraploidy process. (A) Rats from the same litter were weaned at 19 days of age; half of the rats were assigned to a high-carbohydrate (HC) diet and the other half to a high-fat (HF) diet. Before sacrifice on day 25 , rats were fasted for 24 hours and then refed their respective diets for 18 hours. (B) Fed blood glucose and serum insulin concentrations were measured in all animals before sacrifice. Results are mean \pm SEM for at least 8 animals per group. (C) Total RNA was extracted from rat livers ( $n=5$ [high carbohydrate]; 9 [high fat]) and analyzed by quantitative real-time PCR to estimate the abundance of SREBP-1C, L-PK, and FAS mRNAs. Values are normalized to that for cyclophilin $\mathrm{mRNA},{ }^{* *} P<0.01$. (D) Protein extracts were prepared from liver tissue from high-carbohydrate or high-fat diet-fed rats and subjected to immunoblotting with antibodies against SREBP-1c (precursor form; pSREBP-1c), GK, and FAS proteins. $\gamma$-Tubulin was used as a loading control. A representative Western blot is shown. (E) The percentage of binucleated tetraploid cells was calculated as described in Figure $2 \mathrm{C}$. Histogram shows mean \pm SEM of 3 independent experiments $(n=14$ per group). 

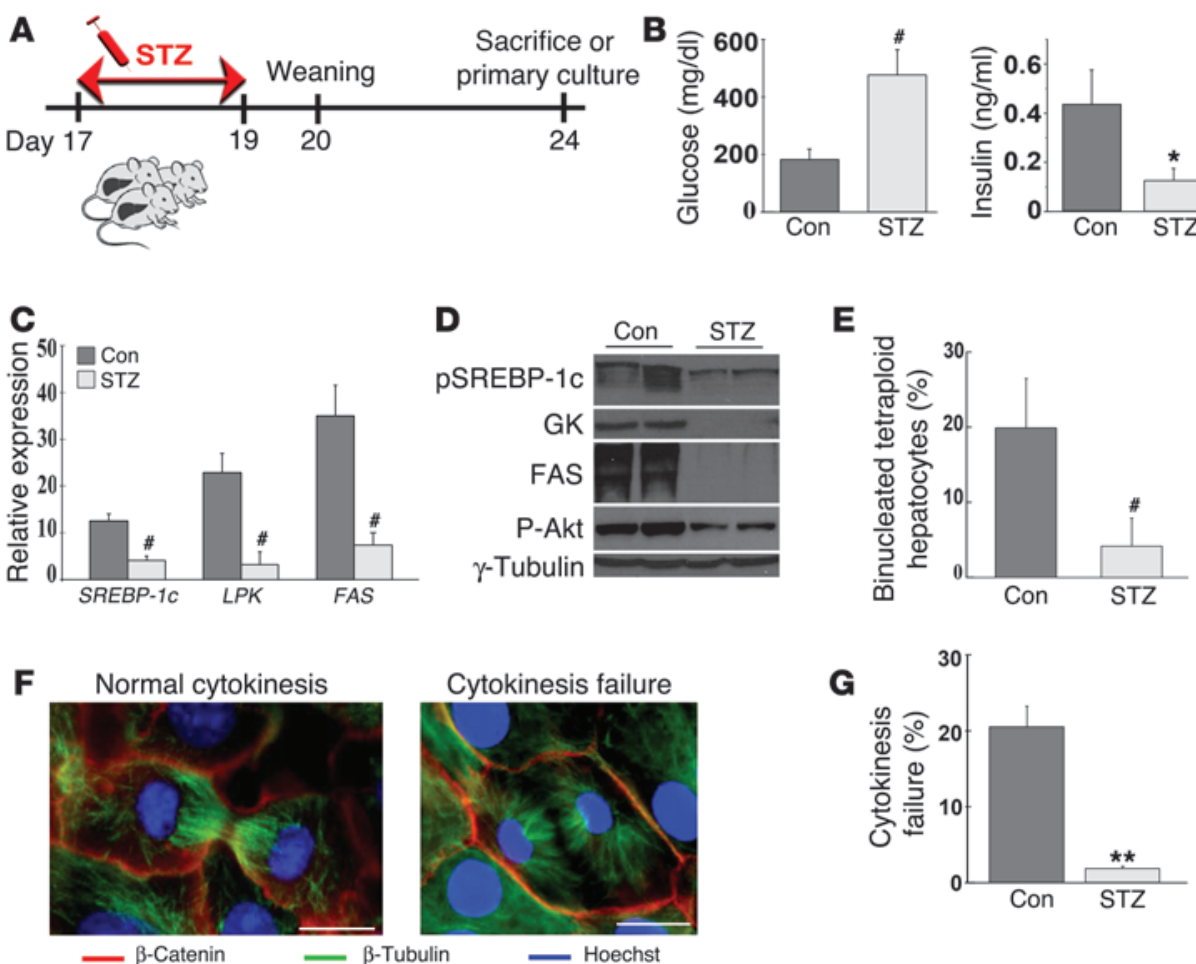

Figure 4

Decreased insulin level abolishes generation of binucleated tetraploid hepatocytes. (A) Rats from the same litter were injected with STZ or vehicle, weaned at 20 days of age, and fed a high-carbohydrate diet. After a postprandial phase, rats were sacrificed at 24 days for in vivo analysis (D) or for isolating primary hepatocytes (E and F). (B) Fed blood glucose and serum insulin concentrations were measured in all animals at 24 days. Results are mean \pm SEM for at least 6 animals per group. Con, control. (C) Total RNA was extracted from control (Con) and STZ-treated rat livers $(n=3)$, and SREBP-1C, $L-P K$, and FAS mRNA levels were analyzed by quantitative real-time PCR. Values are normalized to cyclophilin mRNA. (D) Protein extracts were prepared from liver tissue from control or STZ-treated rats and subjected to immunoblotting with antibodies against the precursor form of SREBP-1c, GK, FAS, and phospho-Akt (Ser473) (P-Akt). $\gamma$-Tubulin was used as a loading control. A representative Western blot is shown. (E) Percentage of tetraploid cells. Histogram shows mean \pm SEM of 3 independent experiments ( $n=6$ [control]; 15 [STZ]). (F) Primary hepatocytes isolated from control and STZ-treated rats were immunostained for $\beta$-catenin, $\beta$-tubulin, and Hoechst for analysis of normal cytokinesis and cytokinesis failure. Scale bars: $10 \mu \mathrm{m}$. (G) Percentage of cytokinesis failure events for rats in F. For each condition, 100 telophases were analyzed. Results are mean $\pm \operatorname{SEM}\left(n=2\right.$ per group). ${ }^{*} P<0.05$, ${ }^{\star \star} P<0.01,{ }^{\#} P<0.005$ versus control.

ment, we were able to follow ex vivo the tetraploidization process (22). To confirm our in vivo findings, we used this technique to test whether hepatocytes from STZ-treated animals were able to progress through the cell cycle and complete cytokinesis to generate diploid progeny (Figure 4A). In our culture conditions, 80\% of hepatocytes from control and STZ-treated animals progressed through $S$ phase 48 hours after plating, with the maximal mitotic index occurring 60 hours after plating (data not shown). Cytokinesis was analyzed by triple labeling of nucleus, microtubules, and membranes (Figure 4F). Cytokinesis failure was observed in $1.8 \% \pm 0.3 \%$ of STZ-treated hepatocyte events, a value much lower than the $20.5 \% \pm 2.7 \%$ for control hepatocytes (Figure $4 \mathrm{G}$ ). These findings demonstrate that decreasing the insulin level resulted in almost all hepatocytes engaging in a normal cell cycle and led to generation of diploid progeny. To reinforce these findings, we analyzed the alteration of tetraploidy process in the $o b / o b$ mouse model, in which, like the STZ model, insulin signaling is impaired

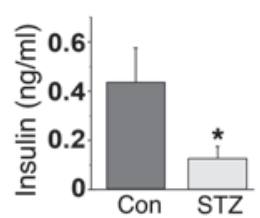

during postnatal liver growth. In the $o b / o b$ model, insulin resistance first starts at the postweaning period and is well established at adult age (28). At 5 weeks after birth, Akt signaling was already impaired in this model (Figure $5 \mathrm{~A}$ ). Furthermore, the percentage of binucleated tetraploid hepatocytes was significantly lower than that in control mice $(25.4 \% \pm 1.8 \%$; Figure 5, $\mathrm{B}$ and $\mathrm{C})$. Together, these results indicate that impairment of insulin signaling inhibits generation of binucleated tetraploid progenies.

Next, we tested whether an increase in the insulin concentration could by itself induce generation of binucleated tetraploid cells. We determined the proliferative capacity of the hepatocytes and their insulin responsiveness during postnatal liver growth by studying the Ki67 labeling index. At days 15-20, just before weaning, proliferation decreased substantially, and the subsequent suckling-toweaning transition beginning at day 20 was associated with an increase in the proliferation index (Figure $6 \mathrm{~A})$. The expression of IR increased specifically after weaning, correlated with the activation of insulin-regulated genes GK and phospho-Akt (Figure 6B). We therefore tested whether an increase in insulin levels at the suckling-to-weaning transition stimulates generation of binucleated tetraploid liver cells. Rats from the same litter were injected with insulin or its vehicle around weaning (Figure 6C). In insulin-treated rats, the level of phospho-Akt was higher than that in control rats (Figure 6D). Remarkably, the proportion of hepatocytes from insulin-treated rats that were tetraploid was $32.2 \% \pm 14.6 \%$ higher than that from control animals (Figure 6E). Together, these observations demonstrate that insulin regulated generation of liver binucleated tetraploid cells.

AKT downstream from insulin signaling regulates generation of binucleated tetraploid hepatocytes. We investigated how insulin controls the cytokinesis program. In our system, cytokinesis failure occurs as a result of the absence of cytoskeleton reorganization, which leads to the inactivation of RhoA signaling (22). The PI3K/Akt pathway appears to be one of the principal downstream pathways mediating the cellular effects of insulin. In particular, signal transduction via the mammalian target of rapamycin (mTOR) has been the subject of intense investigation $(29,30)$. Briefly, mTOR signaling complex 1 (mTORC1) is a key regulator of growth factor and nutrient signaling. S6 kinase is the best-characterized downstream effector of $\mathrm{mTORC} 1$. mTORC2 has a role in regulating the actin cytoskeleton and activating Akt through Ser473 phos- 

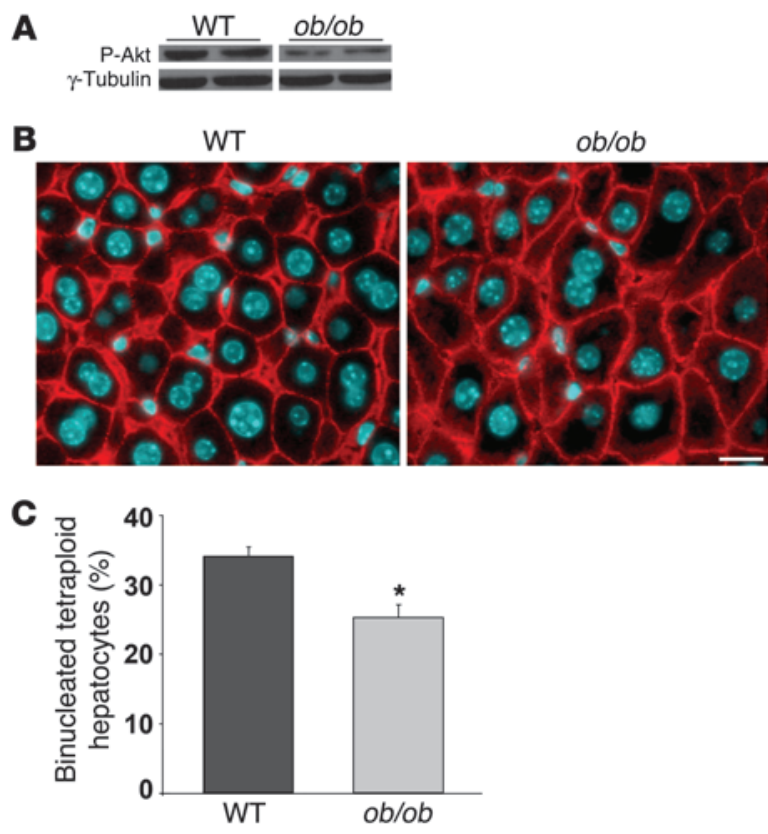

phorylation. We therefore tested whether modulating the PI3K/ Akt pathway affects the cytokinesis program. Primary hepatocytes were isolated from fed rats and treated with specific inhibitors of the PI3K/Akt pathway. Because of the central role played by this pathway at the $G_{1} / S$ transition, pharmacological inhibitors were

\section{Figure 5}

Liver tetraploidy process in ob/ob mice. (A) Immunoblotting with antibody against phospho-Akt (Ser473). $\gamma$-Tubulin was used as a loading control. A representative Western blot is shown $(n=4$ per group). (B) Tissue section after double staining with $\beta$-catenin and Hoechst, illustrating the difference between diploid and tetraploid hepatocytes in 5-week-old WT and ob/ob mice. Scale bar: $10 \mu \mathrm{m}$. (C) Percentage of tetraploid cells in WT and ob/ob mice $(n=4$ per group). ${ }^{*} P<0.05$ versus WT.

added to the culture medium after cells had finished $S$ phase (48 hours after plating) and entered mitosis (60 hours after plating). First we studied the effects of LY294002, a selective PI3K inhibitor, on the cultured hepatocytes. As expected, LY294002 treatment substantially reduced Akt and S6 ribosomal phosphorylation (Figure 7A). Remarkably, this treatment significantly decreased cytokinesis failure events $(46.4 \% \pm 17.1 \%$ fewer than in controltreated animals; Figure 7B). We tested whether the alteration of the MAPK pathway, another major pathway conducting the cellular effects of insulin, affected the cytokinesis program. We found that hepatocytes treated with the MEK inhibitor U0126 presented the same cytokinesis failure rate as untreated control hepatocytes (Figure 7, A and B). To further assess the role of the PI3K pathway, we tested the effects of a specific inhibitor of Akt, iAkt VIII, which prevents Akt phosphorylation (Figure 7A). This direct inhibition of Akt decreased the frequency of cytokinesis failure events by $32.4 \% \pm 8.2 \%$ (Figure $7 \mathrm{~B}$ ). We also examined the cytoskeleton organization in treated cells: iAkt-treated cells that completed

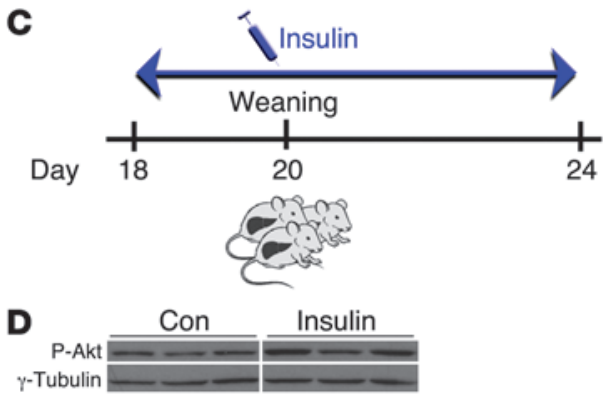

E

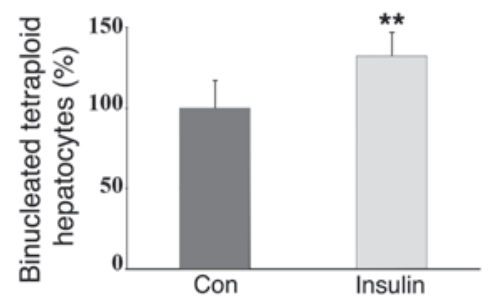

Figure 6

The increase of the circulating insulin concentration around weaning induces generation of binucleated tetraploid liver cells. (A) Proliferative status of hepatocytes during postnatal development (gray, suckling period; pink, feeding period). Tissue sections were immunostained for Ki67. Results are mean \pm SEM ( $n=4$ per time point). (B) Protein extracts were prepared from liver tissues on days 8-25 and subjected to immunoblotting with antibodies against IR, GK, and phospho-Akt (Ser473). $\gamma$-Tubulin was used as a loading control. Shown are a representative Western blot ( $n=5$ per group) and a graph of IR expression. (C) Rats from the same litter were injected with insulin or vehicle during the suckling-to-weaning transition. All rats were weaned at age 20 days, fed a high-carbohydrate diet, and sacrificed at 24 days. (D) Immunoblotting with antibody against phospho-Akt (Ser473). $\gamma$-Tubulin was used as a loading control. A representative Western blot is shown $(n=5$ per group). (E) Percentage of binucleated tetraploid cells, calculated as described in Figure 2C. Histogram shows mean \pm SEM of 3 independent experiments $(n=6)$. ${ }^{* *} P<0.01$ versus vehicle control. 


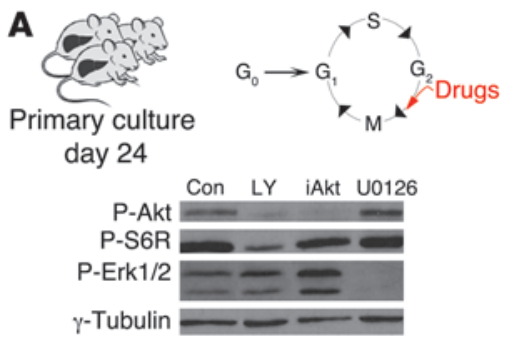

C

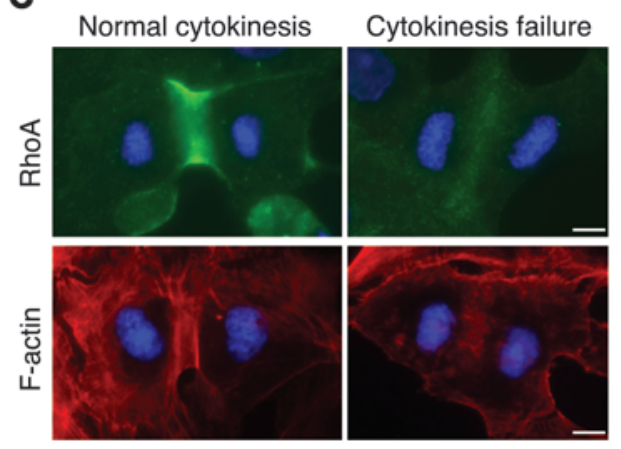

B

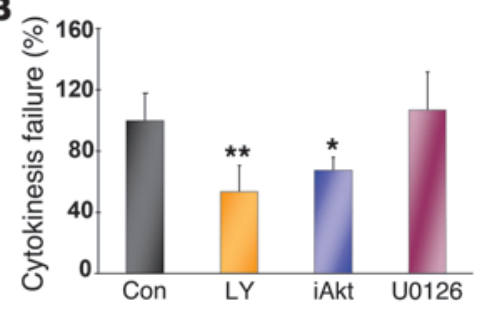

D
E

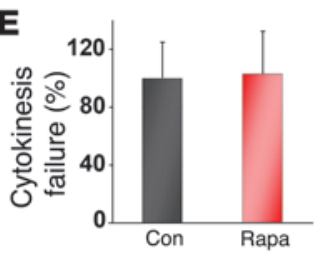

\section{Figure 7}

Akt regulates liver tetraploidy. (A and D) Drugs LY294002 (LY), iAkt VIII (iAkt), U0126, and rapamycin (Rapa) or DMSO control were added to primary hepatocyte cultures of 24-day-old rats 60 hours after plating. Protein extracts were prepared and subjected to Western blotting with antibodies against phospho-Akt (Ser473), phospho-S6 ribosomal protein (Ser240/244; P-S6R), and phosphoErk1/2 (P-Erk1/2). $\gamma$-Tubulin was used as a loading control. Representative Western blots are shown. (B and $\mathbf{E})$ Percentage of cytokinesis failure events in control and treated hepatocytes, calculated by analysis of $\beta$-catenin, $\beta$-tubulin, and Hoechst staining (see Figure 4F). For each condition, 100 telophases were analyzed. Results are representative of 4 independent cultures. (C) Primary hepatocytes rats were immunostained for $\mathrm{F}$-actin, RhoA, and Hoechst for analysis of normal cytokinesis and cytokinesis failure. Scale bars: $5 \mu \mathrm{m}$. ${ }^{*} P<0.05,{ }^{* *} P<0.01$ versus control. cytokinesis reorganized the actin cytoskeleton and recruited RhoA to the equatorial cortex, in contrast to cells that did not complete cytokinesis (Figure 7C). To study the potential role of mTORC1 and mTORC2, primary hepatocytes were treated with rapamycin. As previously described in other cell types $(31,32)$, rapamycin specifically inhibited mTORC1 (phosphorylation of Ser240/244 on S6 ribosomal protein) and not mTORC2 (phosphorylation of Ser473 on Akt; Figure 7D). We observed that rapamycin-treated cells presented the same cytokinesis failure rate as did untreated cells (Figure 7E). This result indicates that mTORC1 does not regulate generation of liver tetraploid cells. The present findings reveal that Akt, directly or indirectly through mTORC2, controlled the cytokinesis program and consequent generation of binucleated tetraploid hepatocytes.

\section{Discussion}

Although most eukaryotic organisms are diploid, many cells have more than 2 chromosome sets. Some mammalian tissues and organs have significant numbers of tetraploid cells that arise during either physiological or pathological growth. Does the polyploid state represent an advantage or a disadvantage for a cell? Interestingly, phenotypic analysis indicates that increasing ploidy can orient cell physiology toward particular functions $(33,34)$. However, generation of polyploid cells can also trigger cell transformation and tumor formation (35). It has been clearly shown that hepatic proliferation both during development and in challenging circumstances, such as after partial hepatectomy or oxidative damage, is associated with ploidization (10). In this context, defining which signals trigger generation of polyploid cells may bring new insight into the physiological properties of these cells and consequently their functions. Here we show, for the first time to our knowledge, that insulin signaling controlled a specific division program that led to generation of binucleated tetraploid liver cells.

We first revealed that cytokinesis failure was controlled by suckling-to-weaning changes. Indeed, nutritional and hormonal changes appearing during this period greatly modify glucose and fatty acid metabolism (23). In parallel to these metabolic changes, we observed a new wave of proliferation in the liver that was associated with the establishment of adaptive cell cycles. Neither the carbohydrate content of the diet nor changes in daily rhythms during this transition appeared to be involved in this specific division program. We demonstrated that insulin, independent of its action on glucose signaling, controlled the extent of cytokinesis failure and thus generation of binucleated tetraploid liver cells. This observation is supported by our finding that when insulin signaling was impaired at weaning, diploid hepatocytes engaged in a cell cycle leading to the generation of diploid progeny. Consistent with this finding, elevation of the circulating insulin concentration was sufficient to induce the appearance of binucleated tetraploid progeny. We thus describe an unforeseen mechanism of action for insulin in the control of cell cycle completion. In the past, extensive studies with yeast, metazoans, and mammals have clearly established that the insulin signaling pathway regulates cell proliferation and growth to create organs of reproducible size and shape (36). Cellular growth and proliferation are coordinated by specific checkpoints in $G_{1}$ or $G_{2}$ phases. Interestingly, a recent study in Drosophila reports that insulin signaling can influence cell number through the balance between its ability to accelerate progression through $\mathrm{G}_{1} / \mathrm{S}$ and delays at the $\mathrm{G}_{2} / \mathrm{M}$ transition (37). Here we demonstrate, for the first time to our knowledge, that insulin also controlled late mitosis progression in mammals by regulating cytokinesis.

The process of cytokinesis can be divided into 4 stages: specification of the cleavage plane, formation and then ingression of the contractile ring, and finally cell abscission (38). Each stage is dependent on the proper execution of the prior stage; thus, interference with any stage may result in cytokinesis failure (39). In the liver, cytokinesis failure occurs as a result of the absence of actin cytoskeleton organization at the cleavage plane. Consequently, active RhoA does not concentrate at the putative site of furrow formation, leading to an absence of activation of its downstream signals (22). How does insulin control this specific cell cycle program? The PI3K/Akt pathway, a mediator of insulin signaling, controls many cellular 
processes, including cytoskeleton organization. In yeast, TORC2 regulates actin cytoskeleton polarization (31); likewise, mTORC2 was previously shown to regulate actin cytoskeleton reorganization $(32,40)$. In mammalian cells, Akt regulates the activity of the ankyrin-repeat domain-containing protein Kank, a central protein involved in RhoA-dependent formation of actin stress fibers and cell migration (41). Recently, it was shown that Akt directly downregulates glycogen synthase kinase-3 (GSK-3) and thus acts on microtubule cytoskeleton during early mitosis (42). Our findings support the concept, which we believe to be novel, that the $\mathrm{PI} 3 \mathrm{~K} /$ Akt pathway is a key actor in the cytokinesis process, which, like cell polarization and migration, requires specific regulation of cytoskeleton networks. Indeed, inhibition of PI3K during mitosis progression prevented cytokinesis failure and consequently committed the cells to divide into diploid progeny. We provide evidence that mTORC1 was not involved in this process. In contrast, direct inhibition of Akt led to the same phenotype as PI3K inhibition and oriented the cell to perform a complete cytokinesis, characterized by actin cytoskeleton reorganization and active RhoA localization to the cleavage plane. Further investigations will be necessary to define whether Akt contributes directly, or in association with mTORC2, to generation of binucleated tetraploid liver cells.

Future studies should investigate why this specific division program emerges under an insulin signal during the suckling-toweaning transition. It is possible that cells escape cytokinesis, a process consuming substantial energy, to respond to this metabolic transition. Alternatively, the tetraploidization process may be required for the cell to engage in particular metabolic functions required at this step for liver development. Interestingly, megakaryocyte polyploidization regulates the gene expression involved in platelet biogenesis (34). Consequently, it would be particularly interesting to compare the metabolic functions of diploid and polyploid liver cells.

In conclusion, our results revealed that modulation of the insulin concentration, and consequently Akt activity, oriented hepatocytes into a specific cell cycle program, leading to generation of binucleated tetraploid cells. Because the pathogenesis of various metabolic liver diseases is associated with major deregulations of the insulin signaling pathway, it may be informative to determine the liver ploidy profile during the development of these types of disorders and consequently whether any ploidy modifications are involved in the pathophysiology of these diseases.

\section{Methods}

Animal experiments. Wistar rats and $o b / o b$ mice (B6.V-Lepob/J; Charles River Laboratories) were housed in a temperature-controlled environment with 12-hour light/12-hour dark cycles and had free access to water and a highcarbohydrate diet (51.7\% of calories from carbohydrate, 5.1\% from fat, and $21.4 \%$ from protein). To modify daily rhythms, cycles were changed from 12-hour light/12-hour dark to 18-hour light/6-hour dark or 21-hour light/3-hour dark. For diet experiments, rats were randomly assigned to be fed the high-carbohydrate diet or the high-fat diet (1.0\% of calories from carbohydrate, $71.8 \%$ from fat, and $28.2 \%$ from protein). Before sacrifice, the 2 groups were fasted for 24 hours and then refed the same diet for 18 hours. For STZ treatment, rats received 1 intraperitoneal injection of STZ (60 mg/kg in $0.1 \mathrm{M}$ citrate buffer, $\mathrm{pH} 4.5)$ on each of days 17,18 , and 19 of age. Controls received vehicle. Human insulin (Insulatard; Novo Nordisk) was administered by intraperitoneal injection at $5 \mathrm{U} / \mathrm{kg}$ body mass. For all experiments, animals were sacrificed, and the liver was either immediately flash frozen and stored at $-80^{\circ} \mathrm{C}$ or fixed in phosphate-buff- ered $10 \%$ formalin. Blood samples were also collected. Glucose values were determined from whole venous blood using an automatic glucose monitor (GlucoMen GlycÓ; A. Menarini Diagnostics). Insulin levels in serum were measured by ELISA using rat standards according to the manufacturer's guidelines (Mouse/Rat Insulin ELISA; Diagnostics Systems Laboratories Inc). Animal studies described herein were reviewed and approved (agreement no. 75-956) by the Directeur départemental des Services Vétérinaires of the Prefecture de Police de Paris (Paris, France).

Cell cultures. Hepatocytes were isolated from rat livers by 2-step perfusion and were cultured as previously described (21). In these conditions, after liver perfusion with collagenase, hepatocytes enter the cell cycle. Mitogenic treatment with EGF allowed these cells to progress synchronously into $S$ phase at 48 hours after plating, with maximal mitotic index at 60 hours

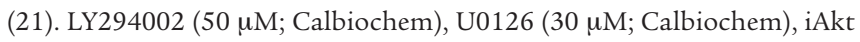
inhibitor VIII ( $1 \mu \mathrm{M}$; Calbiochem), and rapamycin ( $5 \mathrm{nM}$; Sigma-Aldrich) were added 60 hours after plating: 2 hours of treatment for LY294002, U0126, and iAkt and 4 hours of treatment for rapamycin.

Real-time PCR analysis. Total RNA (Macherey-Nagel) was isolated from rat liver and used as a template for cDNA synthesis (Transcriptor First Strand cDNA synthesis; Roche). Quantitative real-time PCR was performed using a LightCycler Instrument (Roche Diagnostics) according to the manufacturer's instructions and with the SYBR green master kit (Roche). Primers used were for cyclophilin (sense, $5^{\prime}$-ATGGCACTGGTGGCAAGTCC-3'; antisense, 5'-TTGCCATTCCTGGACCCAAA- $3^{\prime}$ ), FAS (sense, 5'-ACCTGTCCCAGGTGTGTGAT-3'; antisense, 5'-GCTGTGGATGATGTTGATGA-3'), GK (sense, 5'-CAACATCGTAGGACTTCTCCG-3'; antisense, 5'-GGCGGTCTTCATAGTAGCAG-3'), L-PK (sense, 5'-GTTGTTTGAGGAGCTACGCC-3'; antisense, 5'-GTCTTCGTCAGCACGATGAT- ${ }^{\prime}$ ), and SREBP-1c (sense, $5^{\prime}$-TTGAAGACATGCTTCAGCTC- ${ }^{\prime}$; antisense, $5^{\prime}$-GCCTGTGTCTCCTGTCTCA-3'). The values reported for each mRNA were corrected to the cyclophilin mRNA values.

Western blot. Total protein extracts were obtained from liver samples and primary hepatocyte cultures as previously described (21). Protein extracts were resolved by SDS-PAGE, then transferred onto 0.45 - $\mu \mathrm{m}$ pore-size nitrocellulose membranes. The membranes were incubated overnight at $4^{\circ} \mathrm{C}$ with antibodies against GK (gift from M. Magnusson, Vanderbilt University, Nashville, Tennessee, USA), FAS (gift from I. Dugail, Centre de Recherche des Cordeliers, Paris, France), SREBP-1c (NeoMarkers; Interchim Inc.), phospho-Akt (Ser473) (BD Biosciences), phospho-p44/42 MAPK (Thr202/ Tyr204) (BD Biosciences), phospho-S6 ribosomal protein (Ser240/244) (BD Biosciences), IR $\beta$ (C-19; Santa Cruz Biotechnology Inc.), and $\gamma$-tubulin (GTU88; Sigma). Horseradish peroxidase-conjugated antibodies (GE Healthcare) were used as secondary antibodies. Immunoreactive bands were visualized with a chemiluminescence kit (Thermo Fisher Scientific). The blots were exposed to KODAK Biomax films, and the IR signal was quantified using Genesnap version 6.00 and Genetools version 3.07 software (Syngene). In all Western blot experiments, samples were run on the same gel.

Immunohistochemistry. Liver tissue was fixed overnight in formalin, embedded in paraffin, and cut into $5-\mu \mathrm{m}$-thick slices. Primary hepatocytes were fixed in $-20^{\circ} \mathrm{C} \mathrm{MetOH}$ for 3 minutes (to stain $\beta$-tubulin and $\beta$-catenin), in $4^{\circ} \mathrm{C} 10 \%$ TCA for 15 minutes (to stain RhoA), or in $4^{\circ} \mathrm{C} 4 \%$ PFA for 15 minutes (to stain F-actin). Immunohistochemistry was performed with mouse anti- $\beta$-catenin (diluted 1:200; BD Biosciences), mouse anti- $\beta$-tubulin (diluted 1:400; Tub 2.1; Sigma-Aldrich), mouse antiRhoA (diluted 1:100; Santa Cruz Biotechnology Inc.), and $165 \mathrm{nM}$ Alexa Fluor 488 phalloidin (F-actin; Invitrogen). Anti-mouse IgG antibodies conjugated with Alexa Fluor 488 or Alexa Fluor 594 (diluted 1:500; Invitrogen) were used as secondary antibodies. Hoechst $33342(0.2 \mu \mathrm{g} / \mathrm{ml}$; Sigma-Aldrich) was included in the final wash to counterstain nuclei. For Ki67 labeling, a monoclonal antibody against Ki67 proliferation antigen 
(diluted 1:400; clone KiS5; DAKO) was used. Goat anti-mouse conjugated to horseradish peroxide-labeled polymer (EnVision; DAKO) was used as a secondary antibody. Slides were developed with 3-3'-diaminobenzidine chromogen and counterstained with Gill hematoxylin.

Image acquisition and analysis. Images were taken using a Nikon Statif Eclipse E600 microscope with $\times 40$ magnification, 1.4-0.7 NA PL-APO objectives, a DXM1200 cooled CCD camera (Nikon), and ACT-1 (version 2.63; Universal Imaging). For diploid and tetraploid hepatocyte counting experiments, at least 10 randomly chosen fields of liver tissue were imaged (more than 2,500 cells analyzed). For each field, 2 different focal planes of Hoechst staining were acquired to visualize binucleated tetraploid hepatocytes correctly. For $\beta$-tubulin staining on liver sections and F-actin/ RhoA staining on fixed cells, $z$ axis stacks were collected using a piezoelectric device mounted at the base of a $\times 63$-magnification, 1.4 NA PL-APO objective on a Zeiss DMRA2 microscope with a Coolsnap HQ camera controlled by Metamorph software (version 7.1; Universal Imaging). A total of 20-30 planes (0.2- $\mu \mathrm{m}$-thick slices) was captured for each cell and compiled as single 2-dimensional projections using ImageJ software (http://rsbweb. nih.gov/ij/). All images were imported into Photoshop (version CS; Adobe) for contrast manipulation and figure assembly.

Statistics. All experiments were performed at least 3 times. Results are expressed as mean \pm SEM. Mann-Whitney and Student's 2-sample $t$ tests were used for statistical analyses. A $P$ value less than 0.05 was considered significant.

1. Otto, S.P., and Whitton, J. 2000. Polyploid incidence and evolution. Annu. Rev. Genet. 34:401-437.

2. Gupta, S. 2000. Hepatic polyploidy and liver growth control. Semin. Cancer Biol. 10:161-171.

3. Brodsky, W.Y., and Uryvaeva, I.V. 1977. Cell polyploidy: its relation to tissue growth and function. Int. Rev. Cytol. 50:275-332.

4. van der Heijden, F.L., and James, J. 1975. Polyploidy in the human myometrium. Z. Mikrosk. Anat. Forsch. 89:18-26.

5. Hixon, M.L., et al. 2000. Cks1 mediates vascular smooth muscle cell polyploidization. J. Biol. Chem. 275:40434-40442.

6. Vliegen, H.W., et al. 1995. Polyploidy of myocyte nuclei in pressure overloaded human hearts: a flow cytometric study in left and right ventricular myocardium. Am. J. Cardiovasc. Pathol. 5:27-31.

7. Auer, G.U., Backdahl, M., Forsslund, G.M., and Askensten, U.G. 1985. Ploidy levels in nonneoplastic and neoplastic thyroid cells. Anal. Quant. Cytol. Histol. 7:97-106.

8. Storchova, Z., and Pellman, D. 2004. From polyploidy to aneuploidy, genome instability and cancer. Nat. Rev. Mol. Cell Biol. 5:45-54.

9. Ganem, N.J., Storchova, Z., and Pellman, D. 2007. Tetraploidy, aneuploidy and cancer. Curr. Opin. Genet. Dev. 17:151-162.

10. Celton-Morizur, S., and Desdouets, C. 2009. Polyploidization of liver cells. In Polyploidization and cancer. R.Y.C. Poon, editor. Landes Bioscience. Austin, Texas, USA. In press.

11. Seglen, P.O. 1997. DNA ploidy and autophagic protein degradation as determinants of hepatocellular growth and survival. Cell Biol. Toxicol. 13:301-315.

12. Toyoda, H., et al. 2005. Changes to hepatocyte ploi$\mathrm{dy}$ and binuclearity profiles during human chronic viral hepatitis. Gut. 54:297-302.

13. Gorla, G.R., Malhi, H., and Gupta, S. 2001. Polyploidy associated with oxidative injury attenuates proliferative potential of cells. J. Cell Sci. 114:2943-2951.

14. Madra, S., Styles, J., and Smith, A.G. 1995. Perturbation of hepatocyte nuclear populations induced by iron and polychlorinated biphenyls in C57BL/ $10 \mathrm{ScSn}$ mice during carcinogenesis. Carcinogenesis. 16:719-727.

\section{Acknowledgments}

The authors thank Hélène Gilgenkrantz, Jean Girard, JacquesEmmanuel Guidotti, Claudia Mitchell, Mario Pende, Catherine Postic, and Nassia Sotiropoulos for their critical evaluation of this manuscript and fruitful discussions; Catherine Postic, Carina Prip-Buus, and Benoit Viollet for tools and advice about the design of experiments; and Eleanor Luce and Mathilde Bernot for help with the experiments. Anti-GST rat GK antibody was a gift from Mark Magnusson. S. Celton-Morizur is a recipient of DIM Région Ile de France "Cardio-vasculaire Diabète Obésité" and received a fellowship from the Agence Nationale pour la Recherche (ANR-05-JCJC-0168-01). This study was supported by grants from INSERM (ANR-05-JCJC-0168-01), la Ligue Comité de Paris (RS09/75-70), and Association pour la Recherche sur le Cancer (ARC-1033).

Received for publication January 21, 2009, and accepted in revised form May 6, 2009.

Address correspondence to: Chantal Desdouets, Institut Cochin, Faculté de Médecine Cochin Port Royal, Département d'Endocrinologie Métabolisme et Cancer, 24 rue du Faubourg Saint Jacques, 75014 Paris, France. Phone: 33-1-44-41-24-39; Fax: 33-1-44-41-24-21; E-mail: chantal.desdouets@inserm.fr.

15. Muramatsu, Y., et al. 2000. Increased polyploid incidence is associated with abnormal copper accumulation in the liver of LEC mutant rat. Res. Commun. Mol. Pathol. Pharmacol. 107:129-136.

16. Sigal, S.H., et al. 1999. Partial hepatectomyinduced polyploidy attenuates hepatocyte replication and activates cell aging events. Am. J. Physiol. 276:G1260-G1272.

17. Taylor, M.V. 2002. Muscle differentiation: how two cells become one. Curr. Biol. 12:R224-R228.

18. Vignery, A. 2008. Macrophage fusion: molecular mechanisms. Methods Mol. Biol. 475:149-161.

19. Hixon, M.L., and Gualberto, A. 2003. Vascular smooth muscle polyploidization - from mitotic checkpoints to hypertension. Cell Cycle. 2:105-110.

20. Ravid, K., Lu, J., Zimmet, J.M., and Jones, M.R. 2002. Roads to polyploidy: the megakaryocyte example. J. Cell. Physiol. 190:7-20.

21. Guidotti, J.E., et al. 2003. Liver cell polyploidization: a pivotal role for binuclear hepatocytes. J. Biol. Chem. 278:19095-19101.

22. Margall-Ducos, G., et al. 2007. Liver tetraploidization is controlled by a new process of incomplete cytokinesis. J. Cell Sci. 120:3633-3639.

23. Girard, J., Ferre, P., Pegorier, J.P., and Duee, P.H. 1992. Adaptations of glucose and fatty acid metabolism during perinatal period and suckling-weaning transition. Physiol. Rev. 72:507-562.

24. Barbason, H., Herens, C., Mormont, M.C., and Bouzahzah, B. 1987. Circadian synchronization of hepatocyte proliferation in young rats: the role played by adrenal hormones. Cell Tissue Kinet. 20:57-67.

25. Matsuo, T., et al. 2003. Control mechanism of the circadian clock for timing of cell division in vivo. Science. 302:255-259.

26. Warden, C.H., and Fisler, J.S. 2008. Comparisons of diets used in animal models of high-fat feeding. Cell Metab. 7:277.

27. Denechaud, P.D., et al. 2008. ChREBP, but not LXRs, is required for the induction of glucose-regulated genes in mouse liver. J. Clin. Invest. 118:956-964.

28. Lindstrom, P. 2007. The physiology of obese-hyperglycemic mice [ob/ob mice]. ScientificWorldJournal. 7:666-685.

29. Bhaskar, P.T., and Hay, N. 2007. The two TORCs and Akt. Dev. Cell. 12:487-502.

30. Wullschleger, S., Loewith, R., and Hall, M.N. 2006. TOR signaling in growth and metabolism. Cell. 124:471-484.

31. Loewith, R., et al. 2002. Two TOR complexes, only one of which is rapamycin sensitive, have distinct roles in cell growth control. Mol. Cell. 10:457-468.

32. Jacinto, E., et al. 2004. Mammalian TOR complex 2 controls the actin cytoskeleton and is rapamycin insensitive. Nat. Cell Biol. 6:1122-1128.

33. Galitski, T., Saldanha, A.J., Styles, C.A., Lander, E.S., and Fink, G.R. 1999. Ploidy regulation of gene expression. Science. 285:251-254.

34. Raslova, H., et al. 2007. Interrelation between polyploidization and megakaryocyte differentiation: a gene profiling approach. Blood. 109:3225-3234.

35. Fujiwara, T., et al. 2005. Cytokinesis failure generating tetraploids promotes tumorigenesis in p53null cells. Nature. 437:1043-1047.

36. Leevers, S.J., and McNeill, H. 2005. Controlling the size of organs and organisms. Curr. Opin. Cell Biol. 17:604-609.

37. Wu, M.Y., Cully, M., Andersen, D., and Leevers, S.J. 2007. Insulin delays the progression of Drosophila cells through G2/M by activating the dTOR/dRaptor complex. EMBO J. 26:371-379.

38. Eggert, U.S., Mitchison, T.J., and Field, C.M. 2006. Animal cytokinesis: from parts list to mechanisms. Annu. Rev. Biochem. 75:543-566.

39. Normand, G., and King, R.W. 2009. Understanding cytokinesis failure. In Polyploidization and cancer. R.Y.C. Poon, editor. Landes Bioscience. Austin, Texas, USA. In press.

40. Sarbassov, D.D., et al. 2004. Rictor, a novel binding partner of mTOR, defines a rapamycin-insensitive and raptor-independent pathway that regulates the cytoskeleton. Curr. Biol. 14:1296-1302.

41. Kakinuma, N., Roy, B.C., Zhu, Y., Wang, Y., and Kiyama, R. 2008. Kank regulates RhoA-dependent formation of actin stress fibers and cell migration via 14-3-3 in PI3K-Akt signaling. J. Cell Biol. 181:537-549.

42. Buttrick, G.J., and Wakefield, J.G. 2008. PI3-K and GSK-3: Akt-ing together with microtubules. Cell Cycle. 7:2621-2625. 